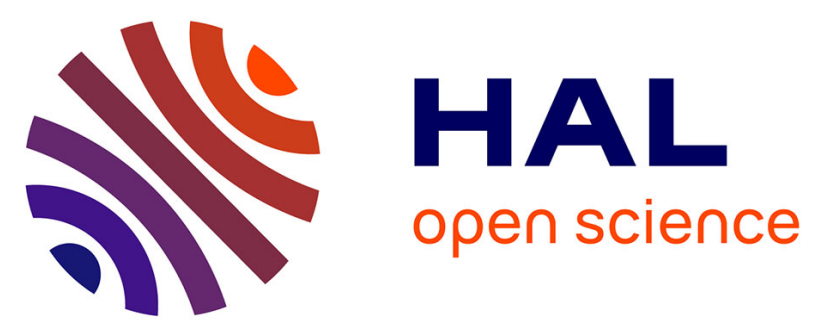

\title{
Electron spin resonance of single iron phthalocyanine molecules and role of their non-localized spins in magnetic interactions
}

Xue Zhang, Christoph Wolf, Yu Wang, Hervé Aubin, Tobias Bilgeri, Philip Willke, Andreas Heinrich, Taeyoung Choi

\section{To cite this version:}

Xue Zhang, Christoph Wolf, Yu Wang, Hervé Aubin, Tobias Bilgeri, et al.. Electron spin resonance of single iron phthalocyanine molecules and role of their non-localized spins in magnetic interactions. Nature Chemistry, 2021, 10.1038/s41557-021-00827-7 . hal-03447967

\section{HAL Id: hal-03447967 https://hal.science/hal-03447967}

Submitted on 24 Nov 2021

HAL is a multi-disciplinary open access archive for the deposit and dissemination of scientific research documents, whether they are published or not. The documents may come from teaching and research institutions in France or abroad, or from public or private research centers.
L'archive ouverte pluridisciplinaire HAL, est destinée au dépôt et à la diffusion de documents scientifiques de niveau recherche, publiés ou non, émanant des établissements d'enseignement et de recherche français ou étrangers, des laboratoires publics ou privés. 
1 Electron spin resonance of single iron-phthalocyanine molecules and role of their 2 non-localized spins in magnetic interaction

3

4 Xue Zhang ${ }^{1,2}$, Christoph Wolf ${ }^{1,2}$, Yu Wang ${ }^{1,2}$, Hervé Aubin ${ }^{3}$, Tobias Bilgeri ${ }^{4}$, Philip Willke ${ }^{1,2,5}$, Andreas J.

$5 \quad$ Heinrich ${ }^{1,6 *}$ and Taeyoung Choi $^{1,6 *}$

6

1 Center for Quantum Nanoscience, Institute for Basic Science (IBS), Seoul 03760, Republic of Korea 2 Ewha Womans University, Seoul 03760, Republic of Korea

3 Universités Paris-Saclay, CNRS, Centre de Nanosciences et de Nanotechnologies, 91120, Palaiseau, France 4 Institute of Physics, École Polytechnique Fédérale de Lausanne, Station 3, CH-1015 Lausanne,

\section{Switzerland}

5 Karlsruhe Institute of Technology, Karlsruhe 76131, Germany

6 Department of Physics, Ewha Womans University, Seoul 03760, Republic of Korea

14

\section{Abstract}

Electron spin resonance (ESR) spectroscopy is a crucial tool in investigations of the chemical structure of materials, and of the electronic structure of materials associated with unpaired spins, through spin-labelling. ESR spectra measured in molecular systems, however, are established on large ensembles of spins and usually require complicated structural analysis. Recently, the combination of scanning tunneling microscopy (STM) with ESR has proven to be a powerful tool to image and coherently control individual atomic spins on surfaces. Here, we extend this technique single coordination complexes - iron phthalocyanines $(\mathrm{FePc})$ - and investigate the magnetic interactions between their molecular spin with either another molecular spin (in FePcFePc dimers) or an atomic spin (in FePc-Ti pairs). We show that the molecular spin density of 
$1 \mathrm{FePc}$ is both localized at the central $\mathrm{Fe}$ atom and also distributed to the outer ligands, yielding a

2 strongly molecular, geometry-dependent exchange coupling.

$4 \quad$ Main text

$5 \quad$ Introduction

6 Chemical engineering and fabrication of single molecular spins is of vital importance in 7 molecule-based quantum devices. ${ }^{1}$ To detect and control single molecular spins, there have been

8 various approaches such as optical detection of diluted molecular spins ${ }^{2-4}$, magnetic resonance 9 force microscopy ${ }^{5}$, nitrogen-vacancy magnetometry ${ }^{6}$, and break junction-based molecular

10 devices $^{7-9}$. Nevertheless, these systems typically require embedding the molecule in a solid-state

11 host and lack the flexibility to locate and access individual spins, or harness intra- and inter12 molecular spin-spin interactions.

13 Conventional electron spin resonance (ESR) studies on chemical ensembles ${ }^{10,11}$ are a very 14 useful tool to elucidate the chemical structures at the molecular level, but they rely on the order of $15 \sim 10^{10}$ spins and often require complementary analysis techniques or theoretical calculations.

16 Recent development of scanning tunneling microscopy (STM) combined with ESR have provided

17 the advantage to investigate single molecular spins with atomic resolution. ESR-STM introduces

18 a radio frequency (rf) electric field at the tunneling junction which can coherently drive individual

19 atomic spins on surfaces ${ }^{12-14}$. The change in spin state is read out by a spin-polarized tip through

20 tunneling magnetoresistance. This combined ESR-STM approach enables precise atom

21 manipulation and imaging in sub-nanometer scale with sub-microelectronvolt $(\sim 10 \mathrm{MHz})$ energy

22 resolution. Previous ESR-STM studies have focused on the spins of single transition metal

23 adatoms $^{12,14-16}$ rather than those of single molecules, besides early attempts on the organic 
1 molecule $-\alpha, \gamma$-bisdiphenylene $\beta$-phenylallyl (BDPA) at room temperature and in ambient

2 condition ${ }^{17}$. Coordination compounds in particular often have non-trivial spin distribution owing

3 to the ligands ${ }^{18}$, giving the unique opportunity to tune the spin distribution on the ligands for

4 engineering spin-spin interaction with atomic resolution.

5 Metal phthalocyanines with various substitutional central metal atoms have been extensively

6 employed as model systems in many-body quantum physics ${ }^{18,19}$ and spin-dependent transport ${ }^{20}$.

7 Here, we explore a system of spin-1/2 iron phthalocyanine (FePc) molecules, iron (Fe) atoms and

8 titanium ( $\mathrm{Ti}$ ) atoms adsorbed on a bilayer magnesium oxide $(\mathrm{MgO})$, itself grown atop a $\operatorname{Ag}(100)$

9 surface. We have performed ESR on individual FePc molecules and characterized the effects of

10 ligand orientations on spin-spin interactions in FePc-FePc dimers and FePc-Ti pairs. This enables

11 us to visualize the electronic configuration of molecular orbitals and characterize the role of the

12 molecular ligands using nearby atoms as sensors, measuring magnetic dipolar and exchange

13 coupling between the molecular spin and ESR-active species (FePc and Ti). DFT calculations, in

14 good agreement with the experimental results, suggest that the spin density of the compound

15 mainly located on the Fe centere spreads to the outer molecular ligands. These findings highlight

16 the role of non-localized spins in the transfer of magnetic interactions, which can be crucial for 17 fabricating molecular devices ${ }^{21}$.

\section{Results and discussion}

20 Single-molecule ESR. Individual FePc molecules, Fe atoms, and Ti atoms were deposited in a 21 step-wise manner onto a bilayer (2-ML) $\mathrm{MgO}$ surface on $\mathrm{Ag}(100)$ substrate and all measurements 22 were performed at a temperature of $2 \mathrm{~K}$ in a commercial STM with vector magnetic fields. The 23 molecules and atoms are well isolated from each other at a low coverage and can be distinguished 
1 readily by their topographical appearance, as shown in Fig. 1a. Individual FePc molecules appear

2 as a cross-like shape and lattice analysis indicates that the central $\mathrm{Fe}$ atom of $\mathrm{FePc}$ of the molecule

3 sits atop an oxygen atom of $\mathrm{MgO}$. The molecular axes are rotated by approximately $27^{\circ}$ with

4 respect to the underlying oxygen rows (inset of Fig. 1a). The Ti atoms in our experiment are mainly

5 found on oxygen-oxygen bridge sites (marked as $\mathrm{Ti}_{\mathrm{B}}$ ) and appear taller than the $\mathrm{Fe}$ atoms atop the 6 oxygen-site $22,23$.

7 Previous studies have reported that FePc possesses a spin $S=1$ in bulk and on several 8 surfaces $^{24,25}$. Surprisingly, our differential conductance $(\mathrm{d} I / \mathrm{d} V)$ spectra measured on well-isolated $9 \mathrm{FePc}$ molecules on a $\mathrm{MgO}$ surface show a clear conductance maximum, reminiscent of the Kondo 10 effect at zero bias when no magnetic field is applied, suggesting the FePc spin being $S=1 / 2$ 11 (Supplementary Fig. 1). This agrees with our DFT calculations, suggesting that an electron is 12 transferred from the Ag substrate to the adsorbed FePc and the molecular spin becomes $S=1 / 2$.

13 The spin density of a negatively charged FePc (referred as $[\mathrm{FePc}]^{1-}$ in the following) is plotted in 14 Fig. 1b, indicating that the spin distributes mainly on the central Fe atom and partially extends 15 along the ligands ${ }^{19}$. Further analysis of the frontier orbital indicates that it consists of about $71 \%$ $16 \mathrm{dz}^{2}$ and $29 \%$ contributions from the ligands and other orbitals (Supplementary Information Section $178,10$, and 11$)$.

18 We performed ESR on individual $[\mathrm{FePc}]^{1-}$ molecules using $\mathrm{rf}$ frequency sweeps ${ }^{12,14}$ at a fixed 19 external magnetic field (Fig. 1c). The external magnetic field sets the Zeeman splitting of the $20[\mathrm{FePc}]^{1-}$ spin and the two Zeeman states of $[\mathrm{FePc}]^{1-}$ spin (labeled as $|0\rangle$ and $|1\rangle$, inset of Fig. 1c) 21 can be coherently driven when the frequency of the applied oscillating electric field $V_{\text {rf }}$ matches 22 the Larmor frequency, which is known as the angular frequency of the spin processing around the 
1 external magnetic field axis. The spin Hamiltonian of a single $[\mathrm{FePc}]^{1-}$ spin system can be written 2 as:

$$
H_{0}=2 \mu_{\mathrm{FePc}} \boldsymbol{B} \cdot \boldsymbol{S},
$$

where $\mu_{\mathrm{FePc}}$ is the magnetic moment of FePc. $\boldsymbol{S}$ is the spin operator and the total magnetic field $\boldsymbol{B}$ which sets the Zeeman splitting is a sum of external magnetic field $\left(\boldsymbol{B}_{\text {ex }}\right)$ and tip field $\left(\boldsymbol{B}_{\text {tip }}\right): \boldsymbol{B}=$ $\boldsymbol{B}_{\text {ex }}+\boldsymbol{B}_{\text {tip }}$. When we apply an external magnetic field along the out-of-plane (z) direction, we simplify the external magnetic field and tip field as $B_{\mathrm{z}}$ and $B_{\text {tip }}$. The resonance frequency $f_{0}$ corresponding to the transition between $|0\rangle$ and $|1\rangle$ states is determined by

$$
h f_{0}=2 \mu_{\mathrm{FePc}}\left(B_{\mathrm{z}}+B_{\mathrm{tip}}\right) .
$$

The continuous-wave ESR signal can be detected when the ESR driving Rabi time is comparable to a geometric mean of spin relaxation $\left(T_{1}\right)$ and spin coherence time $\left(T_{2}\right)^{26}$ (see also Supplementary Section 15). Here, Rabi time describes the half-cycle time needed by a two-level quantum system to perform a cyclic behavior between the two quantum levels and is determined by $T_{R a b i}=\frac{1}{\Omega} \propto \frac{1}{V_{\mathrm{rf}}\left\{1\left|S_{x}\right| 0\right\rangle}$, where $\Omega$ is the Rabi rate. While the time scale of $T_{1}$ and $T_{2}$ are given by a system, an optimal selection of the frequency range and $V_{\mathrm{rf}}$, in principle, can allow the measurement of ESR signal on other spin systems. Therefore, the ESR-STM method can be applied to other atomic and molecular systems as long as the above condition $\left(T_{R a b i} \sim T_{1}, T_{2}\right)$ is satisfied. Here, we note that the ESR peak intensity and linewidth are associated with the spin relaxation time $\left(T_{1}\right)$, spin coherence time $\left(T_{2}\right)$ and driving Rabi rate $\left(\Omega \propto V_{\mathrm{rf}}\right)$. The low bound of $T_{2}$ can be extracted by measuring the ESR peak intensity and the linewidth as a function of $V_{\mathrm{rf}}{ }^{14}$, which is approximately $10 \mathrm{~ns}$ in our case (see Supplementary Section 15). 
As shown in Fig. $1 \mathrm{c}$ and $\mathrm{d}, f_{0}$ of a single FePc molecule shifts linearly to lower frequencies as the set tunneling current $\left(I_{\text {set }}\right)$ increases at fixed DC bias $(V)$ and $B_{z}$, indicating that the tip field 3 ( $\left.B_{\text {tip }}\right)$ is proportional to $I_{\text {set }}^{27,28}$ and opposite to the external magnetic field direction. This holds

4 for all the tips used throughout this paper. In Fig. 1d, the vertical intercept, which is determined by 5 extrapolating the curve of $f_{0}$ vs $I_{\text {set }}$ to zero $B_{\text {tip }}\left(I_{\text {set }}\right)$, corresponds to the Zeeman splitting in the 6 absence of a tip magnetic field. We can thus extract the magnetic moment of an individual [FePc $]^{1-}$ 7 molecule $\left(\mu_{\mathrm{FePc}}\right), 1.058 \pm 0.003 \mu_{\mathrm{B}}$, free from the influence of any tip field. For a given tip at each 8 set tunneling current, $B_{\text {tip }}$ can be calculated precisely by using $B_{\mathrm{z}}-\frac{h f_{0}}{2 \mu_{\mathrm{FePc}}}$ where the fitted $\mu_{F e P c}$ 9 is used, as shown by the double x-axes of Fig. 1d. We also found that the magnetic moment 10 extracted from varied external fields at a fixed tip field gives consistent $[\mathrm{FePc}]^{1-}$ magnetic moment 11 of approximately $1 \mu_{\mathrm{B}}$ and obtained an averaged $\mu_{\mathrm{FePc}}$ of $1.028 \pm 0.023 \mu_{\mathrm{B}}$ by measuring 14 12 individual $[\mathrm{FePc}]^{1-}$ molecules (Supplementary Fig. 3). Moreover, we noted that $\mu_{\mathrm{FePc}}$ differed by 13 approximately $5 \%$ in two different directions of magnetic field (i.e. $B_{\mathrm{x}}$ and $B_{\mathrm{z}}$, Supplementary Fig.

14 2) and a similar anisotropy in magnetic moment has been found in other systems, e.g. bulk cobalt 15 phthalocyanine $(\mathrm{CoPc})^{29}$ and $[\mathrm{FePc}]^{1-}$ crystals $^{30}$ verified by standard ESR measurements. This 16 anisotropy results from the spin-orbit coupling that mixes the quenched $(L=0)$ ground state with 17 excited states of larger orbital moment. The comparison with those bulk measurements also shows 18 that the averaged value of the g-factor for single $[\mathrm{FePc}]^{1-}$ molecule adsorbed on $\mathrm{MgO} / \mathrm{Ag}$ is smaller 19 and closer to that of a free electron. We note that the difference of g-factor between our work on 20 individual $[\mathrm{FePc}]^{1-}$ molecule and bulk $[\mathrm{FePc}]^{1-}$ studies may be explained by different local 21 environment (ligand field) and Kondo screening ${ }^{31,32}$. A detailed discussion is provided in 22 Supplementary Section 12. Our results indicate that the magnetic moment of $[\mathrm{FePc}]^{1-}$ on $\mathrm{MgO}$ is 
weakly anisotropic having spin-1/2 and the tip field can be utilized as a local magnetic field addressing an individual spin. molecular system, we performed ESR measurements on naturally formed $[\mathrm{FePc}]^{1-}-[\mathrm{FePc}]^{1-}$ dimers (one such dimer is outlined in white in Fig. 1a). We define the configuration of a dimer as $(m, n$ ) by labeling the separation between the two Fe centers in the dimer in increments of the oxygen

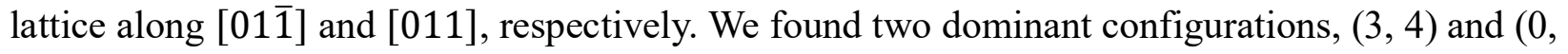
5), which have the identical distance of $1.45 \mathrm{~nm}$ (given the $\mathrm{MgO}$ lattice constant: $0.29 \mathrm{~nm}$ ) between the centers of the two molecules. The occurrence of each configuration is approximately $38 \%$ and $53 \%$, respectively (see all Supplementary Fig. 5). When measuring on one of the $[\mathrm{FePc}]^{1-}$ molecules in either dimer $(3,4)$ or $(0,5)$, we observed multiple ESR peaks as shown in Fig. 2a(ii) and $b($ ii). For each dimer, the splitting between two adjacent ESR peaks appears to be independent

14 of the tip field, implying the splitting is purely associated with the intermolecular coupling while thoroughly discussed in Methods section. The presence of exchange and dipolar coupling terms

21 (denoted as parameters $J$ and $D$, respectively) in the two-spin model makes the quantum eigenstates deviate from the four pure Zeeman product states $|00\rangle,|01\rangle,|10\rangle$ and $|11\rangle$, giving rise to singlet-triplet states. While $|00\rangle$ and $|11\rangle$ remain as the eigenstates of $H_{12}$, the other two 


$$
|-\rangle=-\frac{\alpha}{\sqrt{\alpha^{2}+1}}|01\rangle+\frac{1}{\sqrt{\alpha^{2}+1}}|10\rangle,
$$

$$
|+\rangle=\frac{1}{\sqrt{\alpha^{2}+1}}|01\rangle+\frac{\alpha}{\sqrt{\alpha^{2}+1}}|10\rangle
$$

3 where $\alpha$ indicates the relative weight of $|01\rangle,|10\rangle$ component in the $|-\rangle$ and $|+\rangle$ states $^{22,33}$ and

4 equals to $\frac{\delta+\sqrt{\varepsilon^{2}+\delta^{2}}}{\varepsilon}$, in which $\delta=2\left(\mu_{1}-\mu_{2}\right) B_{\text {ex }}+2 \mu_{1} B_{\text {tip }}$ and $\varepsilon=J-\frac{D}{2}\left(1-3 \cos ^{2} \theta\right) . \mu_{1}$ and

$5 \quad \mu_{2}$ represent the magnetic moment of the $[\mathrm{FePc}]^{1-}$ molecule under the tip and the one apart from

6 the tip, respectively. $B_{\text {ex }}$ and $B_{\text {tip }}$ are external magnetic field and tip field. $\theta$ is the angle between

$7 \hat{\boldsymbol{r}}$ and $[\mathrm{FePc}]^{1-}$ spin orientation. Figure $2 \mathrm{~g}$ depicts a schematic diagram of eigenenergies for the 8 given eigenstates $|00\rangle,|-\rangle,|+\rangle$ and $|11\rangle$ (denoted as $E_{00}, E_{-}, E_{+}$and $E_{11}$ ) as a function of $B_{\mathrm{ex}}$

9 and $B_{\text {tip. }}$. The four possible transitions between these states account for the ESR peaks shown in

10 Fig. 2a(iii) and b(iii), labeled as $f_{1}=\frac{E_{-}-E_{00}}{h}, f_{2}=\frac{E_{11}-E_{+}}{h}, f_{3}=\frac{E_{+}-E_{00}}{h}$, and $f_{4}=\frac{E_{11}-E_{-}}{h}$,

11 respectively. Fitted ESR transitions based on this singlet-triplet model (dotted white curves in Fig.

$12 \mathrm{a}(\mathrm{iii}), \mathrm{b}(\mathrm{iii})$ ) show excellent agreement with the experimental data (Supplementary Information

13 Section 5).

From the expression of $\alpha$ with $\delta$, we note that the tip field can tune the relative weight of $|01\rangle$ and $|10\rangle$ in the $|-\rangle$ and $|+\rangle$ states at given $B_{\mathrm{ex}}$, leading to different dominant ESR transitions at different tip fields (bold arrows in Fig. 2c at weak and strong tip fields). This accounts for the changes in the relative peak intensities of four ESR transitions. In particular, when $B_{\text {tip }}$ is adjusted to achieve $\delta=0$ (occurring at the avoided level crossing indicated by the dashed square in Fig.

$192 \mathrm{c})$ and $\alpha=1$, meaning $B_{\text {tip }}$ compensates the difference of Zeeman energy of two [FePc] ${ }^{1-}$ spins 20 (originated from the unequal magnetic moments), $|10\rangle$ and $|01\rangle$ are equally weighted in $|-\rangle$ and 
$1 \quad|+\rangle, f_{1}$ and $f_{4}$ have the same intensity and $f_{2}, f_{3}$ merge to one peak (when $D \ll J$ and thus negligible), as indicated by the white arrows in Fig. 2a(iii) and b(iii).

Using the above Hamiltonian model, we found that the ESR splitting $\Delta f\left(\Delta f=f_{2}-\right.$

$\left.4 f_{1}=f_{4}-f_{3}\right)$ corresponds to the total coupling energy of the two-spin system,

$$
\Delta f=J+D\left(1-3 \cos ^{2} \theta\right) .
$$

Here, $\Delta f$ depends on the angle between the external field and the sample plane due to an

7 anisotropic dipolar distribution. We recorded $\Delta f$ measured on a $(3,4)$ dimer with rotating external fields in order to differentiate the magnetic dipole interaction from the exchange interaction, as

9 shown in Fig. 2d. By fitting to equation (4), we extracted $J$ and $D$ as $133 \pm 4 \mathrm{MHz}$ and $16 \pm 3 \mathrm{MHz}$, respectively, which strongly suggests that the exchange coupling is the dominant interaction in the

11 molecular spin pairs. The positive sign of $J$ indicates that the coupling is antiferromagnetic (AFM).

12 Moreover, we note that the measured dipolar coupling energy here is consistent with that expected

13 from two ideal atomic spins having $1 \mu_{\mathrm{B}}$ with same center-center distance ${ }^{22,33,34}$. Based on this, we

14 can extract the exchange coupling energy $(J)$ of $[\mathrm{FePc}]^{1-}$ dimers by subtracting the dipolar coupling energy $(D)$ of an atomic dimer with same center-center distance from the measured ESR splitting $16(\Delta f)$.

Previous ESR-STM studies on spin-spin interaction for atomic spins have shown that the 18 exchange coupling is determined exclusively by the interatomic distance, implying that the atomic 19 spin can be treated as a point magnet $22,33,34$. However, we found that the exchange coupling energy 20 of $(3,4)$ and $(0,5)$ molecular dimer are different despite having the same center-center distance. 21 By measuring the ESR splitting $(\Delta f)$ of approximately 20 dimers with $B_{\mathrm{z}}$ field only, we obtained 22 a mean $\Delta \bar{f}$ of $134 \pm 19 \mathrm{MHz}$ for the $(3,4)$ dimer while $64 \pm 7 \mathrm{MHz}$ for the $(0,5)$ dimer 23 (Supplementary Fig. 5). As mentioned above, the corresponding exchange coupling energy $(J)$ 
1 extracted from $\Delta \bar{f}$ is $117 \pm 19 \mathrm{MHz}$ and $47 \pm 7 \mathrm{MHz}$, respectively, by subtracting a dipole

2 contribution of $17 \mathrm{MHz}$ (calculated with two atomic spins having $1 \mu_{\mathrm{B}}$ for each). The non-

3 negligible spin density on the outer ligands of the molecule may produce such a difference by

4 generating additional coupling path for the intermolecular magnetic interaction. This implies that

5 the distance between nearby ligands plays a crucial role in determining the magnetic coupling

6 energy of molecular spin systems.

8 DFT model. We performed DFT calculations to evaluate the influence of the ligand-ligand

9 distance on the exchange coupling energy of $[\mathrm{FePc}]^{1-}$ dimers. For both $(3,4)$ and $(5,0)$ dimers, the

10 optimized adsorption configuration on a $\mathrm{MgO}$ surface was achieved when the two Fe centers are

11 atop oxygen sites and the molecular lobes align along the $(2,1)$ lattice direction, in good agreement

12 with our STM topographic images displayed in Fig. 2(a,i) and 2(b.i). In subsequent calculations,

13 we fixed one molecule in its optimized configuration (lower one in Fig. 3a) while rotating the other

14 molecule (upper one in Fig. 3a) around its Fe center by an angle of $\phi$ with respect to the $(2,1)$

15 lattice direction. The center-center distance remains unchanged during such a rotation while the

16 minimal ligand-ligand distance $d_{\min }$ (defined as the nearest distance between two hydrogen atoms

17 in the benzene rings as shown in Fig. 3c) changes accordingly. The variation in $d_{\min }$ when $\phi$

18 changes in $(3,4)$ and $(5,0)$ configuration is exhibited in the inset of Fig. $3 \mathrm{~b}$. We note a small

19 difference in the $d_{\min }$ of $(3,4)$ and $(5,0)$ dimers when they are in optimized configurations (i.e.

$\left.20 \phi=0^{\circ}\right)$. We then calculated the energy difference between ferromagnetic $\left(E_{\mathrm{FM}}\right)$ and

21 antiferromagnetic coupling $\left(E_{\mathrm{AFM}}\right)$, the value of which equals to $\frac{1}{2} J$ for a two spin- $1 / 2$ system $^{35}$, as

22 a function of $d_{\min }$ (Fig. 3b). The calculated $\left|E_{\mathrm{FM}}-E_{\mathrm{AFM}}\right|$ obeys an exponential decay $(\propto$ 23 $\left.\exp \left(-d_{\min } / d\right)\right)$ as $d_{\min }$ increases, which can be attributed to an exchange interaction through the 
1 molecular ligands. We extract a characteristic decay length of $d=0.0345 \mathrm{~nm}$, which is similar to

2 the length scale of exchange coupling in other molecular system ${ }^{36}$. In our calculations, we note

3 that the exchange coupling energy only differs slightly with and without $\mathrm{MgO}$ substrate, implying

4 the negligible role of the substrate in the intermolecular interaction. Notably, the exchange

5 interaction between two molecular spins is mediated through the fully occupied orbitals of ligand,

6 suggesting a superexchange mechanism (see Supplementary Section 14 for a detailed discussion).

7 The calculated $J$ decreases as $d_{\min }$ gets larger in optimized $(3,4),(0,5)$ and $(2,5)$ dimer

8 configurations, consistent with the trend observed in our experiments (Fig. 3d). Besides, we found

9 a few dimers whose ligand-ligand distance (Supplementary Fig. 7) deviated significantly from the

10 optimized dimer configurations. The exchange coupling energy was significantly larger at equal

11 center-center distance but shorter ligand-ligand distances. This result indicates that the molecular

12 spin-spin coupling can be engineered via tuning inter-ligand symmetry.

14 Sensing molecular spin distribution. To sense the spin distribution on the molecular ligands in greater detail, we substituted a spin-1/2 $\mathrm{Ti}_{\mathrm{B}}$ atom for a FePc molecule and measured the exchange 16 coupling energy in $[\mathrm{FePc}]^{1-}-\mathrm{Ti}_{\mathrm{B}}$ pairs. While $[\mathrm{FePc}]^{1-}$ dimers prefer to arrange only in a few 17 configurations as mentioned above, the relative spatial position of a $\mathrm{Ti}_{\mathrm{B}}$ atom with respect to a $18[\mathrm{FePc}]^{1-}$ molecule can be controlled with atomic precision using atom manipulation. Here, the $\mathrm{Ti}_{\mathrm{B}}$ 19 atom is considered as a point magnet based on previous studies ${ }^{33,34}$ and our DFT calculations 20 (Supplementary Fig. 10). We applied only $B_{\mathrm{Z}}$ field for measuring $[\mathrm{FePc}]^{1-}-\mathrm{Ti}_{\mathrm{B}}$ pairs in order to be 21 able to ignore the angular dependence of the dipolar interaction. The measured exchange coupling 22 energy is thus merely its component along the out-of-plane direction, simplified as J. Figure 4a, c 23 and e show three $[\mathrm{FePc}]^{1-}-\mathrm{Ti}_{\mathrm{B}}$ pairs with the $\mathrm{Ti}_{\mathrm{B}}$ atom sitting on different lattice sites: $(2.5,3),(2.5$, 
3) and $(3.5,1)$ with similar center-center $\left(\mathrm{Fe}^{-T i_{B}}\right)$ distances: $1.13 \mathrm{~nm}, 1.13 \mathrm{~nm}$, and $1.06 \mathrm{~nm}$.

2 However, the relative angle between the connection of $\mathrm{Fe}^{-\mathrm{Ti}_{\mathrm{B}}}$ and the molecular axes varies from

$313.2^{\circ}$ to $42.5^{\circ}$, corresponding to different $\mathrm{Ti}_{\mathrm{B}}$-ligand distances. As the angle becomes larger, i.e.

4 the $\mathrm{Ti}_{\mathrm{B}}$ is further away from the closest $[\mathrm{FePc}]^{1-}$ ligand, we observed a drastic decrease in $J$ by 5 measuring ESR on $[\mathrm{FePc}]^{1-}$, as exhibited in Fig. 4b, d and f. Referring to the $[\mathrm{FePc}]^{1-}-[\mathrm{FePc}]^{1-}$

6 dimer case, the $J$ values shown here is the result after subtracting the dipolar coupling contribution

7 (D) from the ESR splitting $(\Delta f)$. The strong dependence on the relative $\mathrm{Ti}_{\mathrm{B}}$-ligand configuration

8 of the exchange coupling energy indicates that the molecular ligands can influence the magnetic

9 interaction significantly, which agrees with our measurements on $[\mathrm{FePc}]^{1-}-[\mathrm{FePc}]^{1-} \operatorname{dimers}^{-}$

10 To map the molecular geometry-dependent spin distribution of the $[\mathrm{FePc}]^{1-}$ molecules, we 11 measured $J$ of a total of $14[\mathrm{FePc}]^{1-}-\mathrm{Ti}_{\mathrm{B}}$ pairs and observed a drastic decay of $J$ as both $\mathrm{Fe}^{-\mathrm{Ti}_{\mathrm{B}}}$ 12 distance $(r)$ and ligand-Ti ${ }_{\mathrm{B}}$ distance $(l)$ increases (Supplementary Fig. 8). Figure 5 (lower panel) 13 displays the spatial map of $J$ measured in different $[\mathrm{FePc}]^{1-}-\mathrm{Ti}_{\mathrm{B}}$ pairs. Considering the $D_{4 h}$ 14 symmetry of the $[\mathrm{FePc}]^{1-}$ molecule with respect to the $\mathrm{MgO}$ lattice, we duplicated the $J$ of each pair to those equivalent adsorption sites. To emphasize the spin distribution on both Fe center and outer ligands, we utilized a heuristic model that simplified the entire spin distribution as two spin centers sitting at the central Fe atom and the nearest ligand, respectively. In this model, the $J$ of a

$18[\mathrm{FePc}]^{1-}-\mathrm{Ti}_{\mathrm{B}}$ pair with the $\mathrm{Ti}_{\mathrm{B}}$ sitting at $(r, l)$ with respect to the molecule can be described by a sum of two exponential functions, $J(r, l)=J_{0}\left(c_{1} \cdot e^{-r / \lambda}+c_{2} \cdot e^{-l / \lambda}\right)$. Here, $J_{0}$ is the exchange 20 coupling energy constant, which is assumed the same for $\mathrm{Fe}-\mathrm{Ti}_{\mathrm{B}}$ interaction and ligand- $\mathrm{Ti}_{\mathrm{B}}$ 21 interaction. $c_{1}$ and $c_{2}$ are fitting pre-factors. $\lambda$ is the characteristic decay length toward the 22 molecular center $(\mathrm{Fe})$ and the ligand center, respectively. By choosing different lattice sites along 23 the $(2,1)$ direction for determining $l$ in the fitting, we note that $c_{1}$ and $c_{2}$ are optimized when the 
1 reference spot on the ligand is set at $(1.42,0.71)$, giving a ratio of $\frac{c_{2}}{c_{1}}=0.03$ which is consistent with the $<30 \%$ contribution of the spin density from the ligands ( $<7 \%$ for each ligand). The characteristic decay length is fitted as $\lambda=0.0506 \mathrm{~nm}$ (see Supplementary Information Section 7 for more details). This allows us to approximate the spatial distribution of the exchange interaction with a molecule when the $\mathrm{Ti}_{\mathrm{B}}$ spin is positioned at different sites, as shown in the upper panel of Fig. 5. We found good agreement between this simple model and the experimental data by comparing the simulated contour lines with those measured data points, revealing the molecular geometry-dependence of exchange interaction with a molecular spin. These results highlight that the ligands play a crucial role in molecular spin-spin interactions.

In conclusion, we have demonstrated single-molecule ESR by driving the spin of an individual $[\mathrm{FePc}]^{1-}$ molecule on a surface. ESR-STM measurements on molecular dimers and molecule-metal atom pairs enable us to investigate the crucial role of the molecular ligands on the exchange coupling between molecules. The spin-spin interaction energy that we measured is only in hundreds of $\mathrm{MHz}$ range $(\sim 100 \mathrm{neV})$ and the measurement on spin coherence time allows the molecular spin to be one of potential candidates for molecular quantum control experiments. Most importantly, we found that the magnetic exchange interaction with a molecule shows strong dependence on molecular geometry, emphasizing the important role of ligands for the transfer of spin-polarization in molecular systems. Our work extends ESR-STM from single atoms to a much larger class of matter - magnetic molecules. This allows synthetic chemistry to design the spin properties through engineering the ligand field and symmetry. In addition, one can utilize ESRactive atoms or molecules to detect the spin distribution of other general molecular systems via dipolar and exchange interaction. Our work suggests molecules as a potential platform to investigate magnetic interactions with non-localized spins, which has brought intensive interest in 
1 the field of coordination networks and is essential for developing molecule-based spintronic and

2 quantum information devices ${ }^{37}$.

4 Acknowledgements

5 All authors acknowledge support from the Institute for Basic Science under grant IBS-R027-D1.

$6 \quad$ We thank N. Lorente for fruitful discussions.

8 Author Contributions Statement

9 T.C. and X.Z. designed the project. X.Z., Y.W., T.B. and P.W. performed the experiments. C.W.

10 carried out DFT calculations. H.A. contributed to Hamiltonian model simulations. X.Z. and T.C.

11 wrote the manuscript with help of all authors. T.C. and A.J.H. advised the project process. This 12 work is corresponded to A.J.H (heinrich.andreas@qns.science) or T.C.

13 (choi.taeyoung@qns.science).

14

15 Competing Interests Statement

16 The authors declare no competing interests.

18 Figure Legends/Captions

19 Fig. $1 \mid[\mathrm{FePc}]^{1-}$ molecules adsorbed on $\mathrm{MgO} / \mathrm{Ag}(\mathbf{1 0 0})$ and ESR measurements with varied tip fields. a,

20 STM images of $[\mathrm{FePc}]^{1-}$ molecules (light grey cross), Fe (smaller white dot), and $\mathrm{Ti}_{\mathrm{B}}$ (bigger white dot) atoms

21 adsorbed on $\mathrm{MgO}$ surface (dark grey background). A naturally formed [FePc $]^{1-}-[\mathrm{FePc}]^{1-}$ dimer is circled in white.

22 Inset: Magnified view of $[\mathrm{FePc}]^{1-}$ molecules, $\mathrm{Fe}$ and $\mathrm{Ti}_{\mathrm{B}}$ atoms. The blue grids in the inset indicate oxygen sites

23 of the underlying $\mathrm{MgO}$ surface throughout this paper. Black arrows describe the molecular axes pointing to the

24 benzene rings and showing an azimuthal angle of $26.6^{\circ}$ with respect to the oxygen rows. Scanning conditions: 
$V=200 \mathrm{mV}, I_{\text {set }}=12 \mathrm{pA}$ for bigger image and $I_{\text {set }}=10 \mathrm{pA}$ for inset. b, Isosurface representation (in red) of the frontier orbital (isovalue: $10^{-5}$ ) corresponding to the molecular frontier orbital ( $\mathrm{a}_{1 \mathrm{~g}}$ in the inset). Inset: electron occupancy and $d$-orbital components (\%) of the molecular frontier orbitals. $d_{\pi}=0.5\left(d_{z x}+d_{z y}\right)$. Notably, the $\mathrm{a}_{1 \mathrm{u}}$ orbital has no contribution from $d$ component. $\mathbf{c}$, ESR spectra of an individual $[\mathrm{FePc}]^{1-}$ molecule measured at different tunneling currents. ESR conditions: $V=100 \mathrm{mV}, V_{\mathrm{rf}}=10 \mathrm{mV}$. Each spectrum is shifted vertically from one another by $0.75 \mathrm{pA}$ for clarity. Colored lines are Lorentzian fits of raw ESR spectra (black dots). Inset: Schematic representation of switching controlling the electron spin resonance in a two-level system with an rf electric field. d, Linear fit (red line) of resonance frequencies obtained from (c) as a function of $B_{\text {tip }}\left(I_{\text {set }}\right)$, showing the possibility of tuning local field by the tip. Notably, the error bars are smaller than the size of the data dots, thus not distinguishable. The errors shown in (d) originate from the $95 \%$ confidence intervals of the non-linear fits. The source data can be found through the link indicated by Data and Code Availability. PPlease provide source data for this panel]; [Please add error bars].

Fig. 2 | Spin coupling in $[\mathbf{F e P c}]^{1-}-[\mathbf{F e P c}]^{1-}$ dimers. a, b, Dimers in configuration $(3,4)($ a) and $(0,5)(b)$. (i) STM images showing that the $(3,4)$ and $(0,5)$ dimers have the same center-center distance (dashed lines). Scanning conditions: $V=180 \mathrm{mV}, I_{\mathrm{set}}=20 \mathrm{pA}$. (ii) Representative ESR spectra of the upper $[\mathrm{FePc}]^{1-}$ measured at yellow dots in $(\mathbf{a}, \mathbf{i})$ and $(\mathbf{b}, \mathbf{i})$, respectively. (iii) ESR spectra plotted in color scale measured at different $B_{\text {tip }}\left(I_{\text {set }}\right)$, showing clearly the difference in the coupling energy (i.e. $\Delta f)$ of $(3,4)$ and $(0,5)$ dimers. Blue arrows indicate each ESR spectrum presented in (ii). The dotted white curves represent fitted ESR transitions. c, Energy level diagram of a two-spin system considering exchange $(J)$ and dipolar $(D)$ interaction in the presence of $B_{\text {ex }}$ and $B_{\text {tip. }} \mathbf{d}$, Measured (blue dots) and fitted (red curve) ESR splitting $\Delta f$ of a $(3,4)$ dimer while rotating the external field by $\theta$ with respect to the sample plane. The errors and error bars in (iii) and (d) denote the $95 \%$ confidence intervals of the non-linear fits. The total external field $\left|B_{\text {tot }}\right|$ was kept at $550 \mathrm{mT}$ during the field rotation. All ESR spectra were taken with the same tip and $V=100 \mathrm{mV}$. (a) and (b) were obtained with an out-of-plane field 
of $B_{\mathrm{z}}=550 \mathrm{mT}$. $V_{\mathrm{rf}}$ was set as $50 \mathrm{mV}$ for (a,ii), (a.iii), (d) and $40 \mathrm{mV}$ for (b,ii), (b,iii). ESR spectra of the lower $[\mathrm{FePc}]^{1-}$ in each dimer are provided in Supplementary Fig. 4.

Fig. 3 | DFT calculations of exchange coupling in $[\mathrm{FePc}]^{1-}-[\mathrm{FePc}]^{1-}$ dimer atop $\mathrm{MgO}$ only. a, Computational model of a $[\mathrm{FePc}]^{1-}-[\mathrm{FePc}]^{1-}$ dimer. $\mathrm{Fe}-\mathrm{Fe}$ distance is set according to experimental $\mathrm{Fe}$ coordinates. One molecule is fixed while the other one is rotated around the Fe center by $\phi$. The minimum distance between two close-by ligands is denoted as $d_{\min }$ and amplified in (c). b, Calculated energy difference between FM and AFM coupling as a function of $d_{\min }$ for $(3,4)$ (orange dots) and $(0,5)($ blue dots) showing good linearity, indicating a dominant exchange coupling mechanism. Solid lines are fits based on $\left|E_{\mathrm{FM}}-E_{\mathrm{AFM}}\right| \propto \exp \left(-d_{\mathrm{min}} / d\right)$. Inset: $d_{\min }$ as a function of $\phi$. The $d_{\min }$ of optimized $(3,4)$ and $(0,5)$ configurations and corresponding $\left|E_{\mathrm{FM}}-E_{\mathrm{AFM}}\right|$ are highlighted by dashed lines. d, Comparison of calculated (dots) and experimental (triangles) $J$ for three different dimer configurations $((3,4)$, orange; $(0,5)$, blue; $(2,5)$, green), showing very similar trend of $J$ vs $d_{\min }$. The experimental data shown here is only the exchange coupling energy after subtracting the dipolar coupling energy $(17 \mathrm{MHz}$ for the $(3,4),(0,5)$ configurations and $14 \mathrm{MHz}$ for the $(2,5)$ configuration) from the mean ESR splitting $\Delta \bar{f}$. Error bars represent the standard deviation of all measured coupling energies on each dimer configuration with respect to the mean value (see also Supplementary Fig. 5)[Please add a description of the statistical treatment]. ESR spectra of $(2,5)$ dimer are provided in Supplementary Fig. 4.

Fig. 4 | Exchange coupling of $[\mathrm{FePc}]^{1-}-\mathrm{Ti}_{\mathrm{B}}$ pairs with different $\mathrm{Ti}_{\mathrm{B}}$-ligand distance. a, $[\mathrm{FePc}]^{1-}-\mathrm{Ti}_{\mathrm{B}}$ pair with a $(2.5,3)$ configuration. $\mathbf{b}$, A second pair in a $(2.5,3)$ configuration. $\mathbf{c}$, A pair in $(3.5,1)$ configuration. The three pairs have similar $\mathrm{Fe}^{-\mathrm{Ti}_{\mathrm{B}}}$ distances but different angles between molecular axes and the

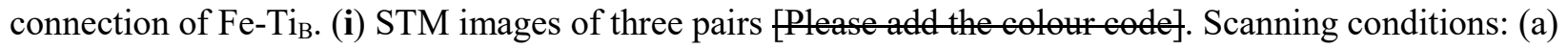
$V=200 \mathrm{mV}, I_{\text {set }}=6 \mathrm{pA}$; (b) $V=200 \mathrm{mV}, I_{\text {set }}=10 \mathrm{pA}$; (c) $V=150 \mathrm{mV}, I_{\text {set }}=20 \mathrm{pA}$. (ii) Respective ESR 
spectra measured on $[\mathrm{FePc}]^{1-}$ (yellow spots in (i)) as a function of tip field by varying setpoint of the

2 tunneling current. The exchange coupling energy $J$ of each $[\mathrm{FePc}]^{1-}-\mathrm{Ti}_{\mathrm{B}}$ pair is labeled on the upper right 3 corner, obtained by subtracting the dipolar coupling contribution (36 MHz for $(2.5,3)$ pair and $44 \mathrm{MHz}$ for

$4(3.5,1)$ pair) from the measured ESR splitting. The significant change in $J$ when $\mathrm{Ti}_{\mathrm{B}}$ is positioned at different

5 sites with respect to the molecular ligands implies a molecular geometry-depended exchange interaction.

6 The errors were obtained from the $95 \%$ confidence intervals of the non-linear fits. The external magnetic field

7 was along out-of-plane direction for all $[\mathrm{FePc}]^{1-}-\mathrm{Ti}_{\mathrm{B}}$ measurements in our work. ESR conditions: $V=100$

$8 \mathrm{mV}$ for all three pairs and (a,ii) $V_{\mathrm{rf}}=30 \mathrm{mV}, B_{\mathrm{z}}=650 \mathrm{mT}$, (b,ii) $V_{\mathrm{rf}}=10 \mathrm{mV}, B_{\mathrm{z}}=600 \mathrm{mT}$, (c,ii) $V_{\mathrm{rf}}=30$

$9 \mathrm{mV}, B_{\mathrm{z}}=570 \mathrm{mT}$.

11 Fig. 5 | Molecular geometry-dependent exchange coupling in $[\mathrm{FePc}]^{1-}-\mathrm{Ti}_{\mathrm{B}}$ pairs. Lower panel: measured

$12 J$ of different $[\mathrm{FePc}]^{1-}-\mathrm{Ti}_{\mathrm{B}}$ pairs expressed by colored dots and labelled at corresponding $\mathrm{Ti}_{\mathrm{B}}$ adsorption sites

13 with respect to the central $[\mathrm{FePc}]^{1-}$ model. In total, 14 pairs were measured and the data points corresponding

14 to the measured $J$ have been equivalently duplicated by a rotation and reflection process according to the

15 molecular symmetry axes (dashed lines). The grey grids represent the oxygen lattice. $J$ is the exchange

16 coupling energy after subtracting the dipole contribution from the total ESR splitting. The contour lines are

17 projected from the simulated spatial exchange coupling energy map choosing those $J$ values obtained from

18 the experiment. Upper panel: simulated spatial exchange coupling energy map by using a sum of two

19 exponential terms representing $r$-dependence and $l$-dependence, respectively. The simulated results are in

20 good agreement with the experimental data, unveiling the geometry-dependence of the exchange coupling

21 with a molecule. The frequency range used in the simulation is set as 3,000 $\mathrm{MHz}$ (upper limit) and $5 \mathrm{MHz}$

22 (lower limit). 
1 [1] Atzori, M. \& Sessoli, R. The second quantum revolution: role and challenges of molecular

2 chemistry. J. Am. Chem. Soc. 141, 11339-11352 (2019).

3 [2] Wrachtrup, J., von Borczyskowski, C., Bernard, J., Orrit, M. \& Brown, R. Optical detection of

4 magnetic resonance in a single molecule. Nature 363, 244-245 (1993).

5 [3] Köhler, J. et al. Magnetic resonance of a single molecular spin. Nature 363, 242-244 (1993).

6 [4] Bayliss, S. L. et al. Optically addressable molecular spins for quantum information processing.

$7 \quad$ Science 370, 1309-1312 (2020).

8 [5] Rugar, D., Budakian, R., Mamin, H. J. \& Chui, B. W. Single spin detection by magnetic

9 resonance force microscopy. Nature 430, 329-332 (2004).

10 [6] Lovchinsky, I. et al. Nuclear magnetic resonance detection and spectroscopy of single proteins

11 using quantum logic. Science 351, 836-841 (2016).

12 [7] Gehring, P., Thijssen, J. M. \& van der Zant, H. S. J. Single-molecule quantum-transport

13 phenomena in break junctions. Nat. Rev. Phys. 1, 381-396 (2019).

14 [8] Vincent, R., Klyatskaya, S., Ruben, M., Wernsdorfer, W. \& Balestro, F. Electronic read-out of

15 a single nuclear spin using a molecular spin transistor. Nature 488, 357-360 (2012).

16 [9] Thiele, S. et al. Electrically driven nuclear spin resonance in single-molecule magnets. Science

$17 \mathbf{3 4 4}, 1135-1138(2014)$.

18 [10] Tesi, L. et al. Quantum coherence in a processable vanadyl complex: new tools for the search 19 of molecular spin qubits. Chem. Sci. 7, 2074-2083 (2016).

20 [11] Graham, M. J. et al. Influence of electronic spin and spin-orbit coupling on decoherence in 21 mononuclear transition metal complexes. J. Am. Chem. Soc. 136, 7623-7626 (2014).

22 [12] Seifert, T. S. et al. Single-atom electron paramagnetic resonance in a scanning tunneling 23 microscope driven by a radio-frequency antenna at 4 K. Phys. Rev. Res. 2, 013032 (2020). 
1 [13] Natterer, F. D. et al. Upgrade of a low-temperature scanning tunneling microscope for

2 electron-spin resonance. Rev. Sci. Instrum. 90, 013706 (2019).

3 [14] Baumann, S. et al. Electron paramagnetic resonance of individual atoms on a surface. Science

$4 \quad 350,417-420(2015)$.

5 [15] Willke, P. et al. Hyperfine interaction of individual atoms on a surface. Science 362, 336-339

6 (2018).

7 [16] Yang, K. et al. Electrically controlled nuclear polarization of individual atoms. Nat.

$8 \quad$ Nanotechnol. 13, 1120-1125 (2018).

9 [17] Durkan, C. \& Welland, M. E. Electronic spin detection in molecules using scanning-

10 tunneling-microscopy-assisted electron-spin resonance. Appl. Phys. Lett. 80, 458-460 (2002).

11 [18] Hiraoka, R. et al. Single-molecule quantum dot as a Kondo simulator. Nat. Commun. 8, 16012 12 (2017).

13 [19] Mugarza, A. et al. Electronic and magnetic properties of molecule-metal interfaces: transition14 metal phthalocyanines adsorbed on $\operatorname{Ag}(100)$. Phys. Rev. B 85, 155437 (2012).

15 [20] Yang, K. et al. Tunable giant magnetoresistance in a single-molecule junction. Nat. Commun. $16 \mathbf{1 0 , 3 5 9 9 ( 2 0 1 9 ) .}$

17 [21] Bogani, L. \& Wernsdorfer, W. Molecular spintronics using single-molecule magnets. Nat. 18 Mater. 7, 179-186 (2008).

19 [22] Bae, Y. et al. Enhanced quantum coherence in exchange coupled spins via singlet-triplet 20 transitions. Sci. Adv. 4, eaau4159 (2018).

21 [23] Willke, P., Yang, K., Bae, Y., Heinrich, A. J. \& Lutz, C. P. Magnetic resonance imaging of 22 single atoms on a surface. Nat. Phys. 15, 1005-1010 (2019). 
1 [24] Tsukahara, N. et al. Adsorption-induced switching of magnetic anisotropy in a single iron(II)

2 phthalocyanine molecule on an oxidized $\mathrm{Cu}(110)$ surface. Phys. Rev. Lett. 102, 167203 (2009).

3 [25] Hiraoka, R. et al. Single-molecule quantum dot as a Kondo simulator. Nat. Commun. 8, 16012

4 (2017).

5 [26] Abragam, A. \& Bleaney, B. Electron Paramagnetic Resonance of Transition Ions (Oxford

6 Univ. Press, 2012).

7 [27] Yang, K. et al. Tuning the exchange bias on a single atom from $1 \mathrm{mT}$ to 10 T. Phys. Rev. Lett.

$8 \quad \mathbf{1 2 2}, 227203(2019)$.

9 [28] Yan, S., Choi, D.-J., Burgess, J. A. J., Rolf-Pissarczyk, S. \& Loth, S. Control of quantum

10 magnets by atomic exchange bias. Nat. Nanotechnol. 10, 40-45 (2015).

11 [29] Assour, J. M. \& Kahn W. K. Electron spin resonance of $\alpha$ - and $\beta$-cobalt phthalocyanine. $J$.

12 Am. Chem. Soc. 87, 207-212 (1965).

13 [30] Konarev, D. V. et al. Ionic compound containing iron phthalocyanine $\left(\mathrm{Fe}^{\mathrm{I}} \mathrm{Pc}\right)^{-}$anions and $14\left(\mathrm{C}_{70^{-}}\right)_{2}$ dimers. Optical and magnetic properties of $\left(\mathrm{Fe}^{\mathrm{I}} \mathrm{Pc}\right)^{-}$in the solid state. Dalton Trans. 41, $15 \quad 13841-13847(2012)$.

16 [31] Wolf, E. L. \& Losee D. L. G-shifts in the "s-d" exchange theory of zero-bias tunneling 17 anomalies. Phys. Lett. A 29, 334-335 (1969).

18 [32] Barnes, S. E. Theory of electron spin resonance of magnetic ions in metals. Adv. Phys. 30, $19 \quad 801-938(1981)$.

20 [33] Yang, K. et al. Engineering the eigenstates of coupled spin-1/2 atoms on a surface. Phys. Rev. 21 Lett. 119, 227206 (2017).

22 [34] Choi, T. et al. Atomic-scale sensing of the magnetic dipolar field from single atoms. Nat. 23 Nanotechnol. 12, 420-424 (2017). 
1 [35] Noodleman, L. Valence bond description of antiferromagnetic coupling in transition metal

2 dimers. J. Chem. Phys. 74, 5737-5743 (1981).

3 [36] Czap, G. et al. Probing and imaging spin interactions with a magnetic single-molecule sensor.

$4 \quad$ Science 364, 670-673 (2019).

5 [37] Ferrando-Soria, J. et al. A modular design of molecular qubits to implement universal quantum 6 gates. Nat. Commun. 7, 11377 (2016).

\section{Methods} STM (Unisoku, USM1300). A radio frequency (rf) microwave which was generated by a signal

11 generator (Keysight, E8257D) was added to a DC voltage $(V)$ using a bias tee at the tip side. With

12 this set-up, an oscillating electric field $V_{\mathrm{rf}}$ was applied at the tunneling junction. The magnetic tip 13 was prepared by transferring 3 5 Fe atoms from $\mathrm{MgO}$ surface to the tip apex and allowed a readout 14 of the spin state by the spin-polarized tunneling current. During an ESR frequency sweep, the rf 15 voltage and DC bias were applied continuously but the rf voltage was modulated at $95 \mathrm{~Hz}$. The modulated tunneling current was then recorded by lock-in technique. All voltages in this paper are

17 referred to the sample.

Sample preparation. In our work, all the materials used for sample preparation were commercially purchased. Single crystalline $\mathrm{Ag}(100)$ was purchased from MaTek. FePc was purchased from Sigma-Aldrich with a purity of $90 \%$. Highly-pure Fe and Ti rods $(99.95 \%)$ were purchased from Goodfellow. $\mathrm{Mg}$ in dendritic pieces was purchased from Sigma-Aldrich with a purity of $99.998 \%$. The $\mathrm{O}_{2}(99.995 \%)$ used here was supplied by a local vendor. The $\operatorname{Ag}(100)$ 
$1 \mathrm{Ag}(100)$ was kept at $400^{\circ} \mathrm{C}$ and magnesium was evaporated onto clean $\mathrm{Ag}$ surface in an $\mathrm{O}_{2}$

2 atmosphere of $1.1 \times 10^{-6}$ torr. FePc was first deposited onto the $\mathrm{MgO}$ surface while the sample was

3 kept at room temperature. The sample was then transferred to the cryostat for cooling down. Fe

4 and $\mathrm{Ti}$ atoms were deposited subsequently onto the cold sample at $25-40 \mathrm{~K}$. After the dosage of

5 molecules and metal atoms, the sample was transferred to $\sim 2 \mathrm{~K}$ environment for ESR

6 measurements. The thickness of the $\mathrm{MgO}$ filmwas determined by performing point-contact

7 measurements on individual Fe atoms ${ }^{38}$ and varied from 2 to 4 monolayers (2-ML to $4-\mathrm{ML}$ ) in our

8 case. Our experiments were all performed on $2 \mathrm{ML} \mathrm{MgO}$. Also, we note that the $\mathrm{MgO}$ patches

9 were found to be surrounded with large irregular Ag terraces. Detailed discussion on the sample

10 morphology is provided in Supplementary Section 9.

11 All the STM images displayed in this work were processed by WSxM" ${ }^{39}$ in "Bone" color mode.

12 A low-order Gaussian filtering function was applied to the images for better visual effect without

13 hindering the authenticity. The original STM files and unprocessed images can be found in Ref.

14 40. All the ESR spectra plotted in color scale were dealt with "Copper" color mode.

Titanium atom manipulation. After titanium deposition, naturally formed $[\mathrm{FePc}]^{1-}-\mathrm{Ti}_{\mathrm{B}}$ pairs were abundant. In addition, we were able to position $\mathrm{Ti}_{\mathrm{B}}$ atom using atom manipulation and construct $[\mathrm{FePc}]^{1-}-\mathrm{Ti}_{\mathrm{B}}$ pairs with various configurations. When the tunneling conductance was set as $V \approx 350 \mathrm{mV}, I \approx 2.2 \mathrm{nA}$, the $\mathrm{Ti}_{\mathrm{B}}$ atom under the tip could follow the tip movement and be positioned at desired sites. In contrast, controllable manipulation of $[\mathrm{FePc}]^{1-}$ molecule rarely occurs under our manipulation parameters.

Hamiltonian model of the two-molecule system. In order to understand the ESR spectra quantitatively, we used a Hamiltonian model of the two-spin system including exchange and dipolar coupling ${ }^{33,34}$ between two FePc molecules: 


$$
H_{12}=2 \mu_{1}\left(\boldsymbol{B}_{\mathrm{ex}}+\boldsymbol{B}_{\mathrm{tip}}\right) \cdot \boldsymbol{S}_{1}+2 \mu_{2} \boldsymbol{B}_{\mathrm{ex}} \cdot \boldsymbol{S}_{2}+J \boldsymbol{S}_{1} \cdot \boldsymbol{S}_{2}+D\left[\boldsymbol{S}_{1} \cdot \boldsymbol{S}_{2}-\mathbf{3}\left(\boldsymbol{S}_{1} \cdot \hat{\boldsymbol{r}}\right)\left(\boldsymbol{S}_{2} \cdot \hat{\boldsymbol{r}}\right)\right]
$$

2 Here, the subscripts 1,2 represent the two $[\mathrm{FePc}]^{1-}$ spins in a dimer. The one under the tip is 3 denoted as 1 whose Zeeman energy is set by both external magnetic field ( $\left.\mathrm{B}_{\mathrm{ex}}\right)$ and tip field ( $\left.\mathrm{B}_{\text {tip }}\right)$.

5 of each $[\mathrm{FePc}]^{1-}$. The first two terms describe the Zeeman energy of a dimer system. Both $[\mathrm{FePc}]^{1-}$ spins align with the external magnetic field direction since both molecules are spin-1/2. The third and last term represent intermolecular exchange and dipolar coupling with the energy constant $J$ and $D$, respectively. $\hat{\boldsymbol{r}}$ is the unit distance vector connecting the centers of two FePc spins. $D$ is given by $\frac{\mu_{0} \mu_{1} \mu_{2}}{\pi r^{3}}$, where $\mu_{0}$ is the vacuum permeability.

DFT calculations. All density functional theory (DFT) calculations were performed using Quantum Espresso (version 6.5) which implements DFT using plane waves and pseudopotentials ${ }^{41,42}$. Pseudopotentials were chosen based on the SSSP library and the basis set was expanded using a kinetic cutoff of $40 \mathrm{Rydberg}^{43}$. All pseudopotentials use the generalized gradient approximation of Perdew, Burke and Ernzerhof (PBE) and we treated van der Waals interaction using Grimme's D3 ${ }^{44,45}$. For single FePc, the calculation model includes $4 \mathrm{ML}$ of silver capped by $2 \mathrm{ML}$ of $\mathrm{MgO}$ exposing the (100) surface. In z-direction, the cell is padded with $1.2 \mathrm{~nm}$ of vacuum. For FePc-FePc dimer, the cell was laterally expanded to accommodate both molecules and make sure that the separation of dimers and their periodic image is at least 5 times larger than the inter-dimer distance. The exchange coupling energy was calculated using the broken-symmetry approach introduced by Noodleman ${ }^{35}$. This approach maps the Kohn-Sham energies of the highspin $\left(m_{s}=1\right)$ and the broken symmetry $\left(m_{s}=0\right)$ state to the diagonal elements of the Heisenberg Hamiltonian. More computational details can be found in Supplementary Information. The 
computational models are provided as Additional Supplementary Files and also available in Ref. 40.

\section{Data and Code Availability}

All data that support the findings of this study are available in this manuscript and its Supplementary Information, or from the corresponding authors on reasonable request. The source data, code and DFT models supporting all figures displayed in main article and supplementary information $^{40}$ are also publicly available through the link https://doi.org/10.6084/m9.figshare.16574534.v1 that directs to Figshare.

\section{References for Methods}

[38] Paul, W. et al. Control of the millisecond spin lifetime of an electrically probed atom. Nat. Phys. 13, 403-407 (2017).

[39] Horcas, I. et al. WSXM: a software for scanning probe microscopy and a tool for nanotechnology. Rev. Sci. Instrum. 78, 013705 (2007).

[40] Zhang, X. et al. Electron spin resonance of single iron-phthalocyanine molecules and role of their non-localized spins in magnetic interaction (source data, codes and raw images). Figshare DOI: 10.6084/m9.figshare.16574534.v1 (2021).

[41] Giannozzi, P. et al. QUANTUM ESPRESSO: a modular and open-source software project for quantum simulations of materials. J. Phys. Condens. Matter 21, 395502 (2009). 
1 [42] Giannozzi, P. et al. Advanced capabilities for materials modelling with Quantum ESPRESSO.

2 J. Phys. Condens. Matter 29, 465901 (2017).

3 [43] Prandini, G., Marrazzo, A., Castelli, I. E., Mounet, N. \& Marzari, N. Precision and efficiency

4 in solid-state pseudopotential calculations. npj Comput. Mater. 4, 1-17 (2018).

5 [44] Perdew, J. P., Burke, K. \& Ernzerhof, M. Generalized gradient approximation made simple.

$6 \quad$ Phys. Rev. Lett. 77, 3865-3868 (1996).

7 [45] Grimme, S., Hansen, A., Brandenburg, J. G. \& Bannwarth, C. Dispersion-corrected mean8 field electronic structure methods. Chem. Rev. 116, 5105-5154 (2016). 